\title{
A DISCRETE-TIME INTERPRETATION OF THE PLANCK-EINSTEIN EQUATION
}

\section{A. BOYARSKY}

Received 2 July 2006; Accepted 21 August 2006

\begin{abstract}
An important conclusion of both string theory and loop quantum gravity theory is that space and time are ultimately discrete. A consequence of discrete space is that there is empty space between the basic elements of space. Analogously, there are empty times between the times where time exists. When time does not exist, it is meaningless to consider the existence of the universe. In this note we consider a discrete-time interpretation of the Planck-Einstein equation and draw a curious conclusion about the real age of the universe.
\end{abstract}

Copyright (c) 2006 A. Boyarsky. This is an open access article distributed under the Creative Commons Attribution License, which permits unrestricted use, distribution, and reproduction in any medium, provided the original work is properly cited.

\section{Introduction}

An important conclusion of both string theory and loop quantum gravity theory is that space and time are ultimately discrete. A consequence of discrete space is that there are gaps between the basic elements of space, that is, space does not exist between the elements of space. Analogously, time does not exist in the interstices between the discretetime elements. When time does not exist it is meaningless to consider the existence of the universe. The notion of existence is not new to physics. For example, it is well known in quantum electrodynamics that virtual particles pop in and out of existence.

In this note we interpret the Planck-Einstein equation from the perspective of discretetime existence, namely, that photons and indeed matter exist only on discrete elements of time. For example, if we consider a fence consisting of discrete, disjoint posts, we can define the total length of the fence-from the first post to the last post including all the spaces between the posts, and we can define the actual length of the posts alone. This distinction applies to time as well; we refer to the time between two events as the total time, and the time of existence as the real time between the two events. 
We associate with light waves and (de Broglie) matter waves a time-dependent energy waveform which reflects the postulate that a photon/particle comes in and out of existence in accordance with the discreteness of time. It then follows that the energy in the Planck-Einstein equation $E=h v$ is an average energy over the total time. This interpretation together with recent estimates for the cutoff in the ultra-high energy cosmic ray spectrum are used to compute the real age of the universe.

\section{Time form for photon and matter waves}

In 1905 Einstein postulated the equation $E=h v$, where $E$ is the energy of a photon with frequency $\nu$ and Planck constant $h=6.626 \ldots \times 10^{-34} \mathrm{~J}$ seconds. In this note we suggest an interpretation of the Planck-Einstein equation that is based on the postulate that photons and matter exist only on part of their wave cycle. In diffraction and interference of waves only the wavelength and amplitude of the wave play a role, not the actual waveform. "In most problems in optics we are concerned only with the amplitude ... of the ... wave $\ldots$ and not with its time variation. This is because the eye and most common measuring instruments respond to the resultant intensity of the light and cannot detect the rapid time variations that characterize light [5]." This can be understood from the fact that the diffraction and interference patterns - even in waves of nonsinusoidal form-are mostly a consequence of the fundamental harmonic which has the same wavelength as the wave itself.

Consider a photon or particle having an associated wave of frequency $\nu$. Then $\nu=v / \lambda$, where $\mathrm{v}$ is the velocity of the wave and $\lambda$ its wavelength. For photons, $\mathrm{v}=c$, the speed of light.

We postulate that the energy waves associated with light and matter have a time dependent form; they exist only on the discrete Planck length intervals, that is,

(P1) during each cycle of the wave, the photon/particle comes in and out of existence;

(P2) when a photon/particle exists (in 4-dimensional space-time) it has a fundamental minimal energy [3, page149], the Planck energy, on Planck units of time. It does not exist when its energy is 0 .

The Planck unit of time is $T_{P}=\left(h G / 2 \pi c^{5}\right)^{1 / 2}=5.39110^{-44}$ seconds, which does not depend on any physical object. It represents an absolute scale, independent of particle. The Planck unit of energy is $E_{P}=\left(h c^{5} / 2 \pi G\right)^{1 / 2}=1.95610^{9} \mathrm{~J}$, which is also independent of particle and scale. The simplest example of (P2) occurs when a photon/particle has energy $E_{P}$ on $N$ contiguous units of Planck times. Let $H=N T_{P}$, which-for the moment-is assumed to be less than the time duration of 1 wavelength, $\lambda / c$.

Let $\operatorname{EX}(t)$ denote the energy wave of light with wavelength $\lambda$, as a function of time:

$$
\operatorname{EX}(t)= \begin{cases}E_{P}, & 0 \leqslant t \leqslant H, \\ 0, & H<t \leqslant \frac{\lambda}{c} .\end{cases}
$$

Let $\bar{E}$ be the average energy over a cycle. Then,

$$
\bar{E}=\frac{1}{\lambda / c} \int_{0}^{\lambda / c} \operatorname{EX}(t) d t
$$


and we obtain

$$
\bar{E}=\frac{1}{\lambda / c} \int_{0}^{H} E_{P} d t=\frac{E_{P} H}{\lambda / c}=E_{P} H \nu .
$$

Letting $E_{P} H=h$, the average energy $\bar{E}=E$ in the Planck-Einstein equation, then, $H=$ $h / E_{P}=3.387710^{-43}$ seconds. To the closest integer, $H$ is 6 Planck times. For the model to be consistent with our assumptions, we require

$$
\frac{\lambda}{c}>H=3.387710^{-43} \mathrm{sec}
$$

or, $\lambda>H c=1.007210^{-34} \mathrm{~m}$. This is an extremely small number, which does not create any physical restriction on wavelengths.

The de Broglie equation states that a particle of mass $\mathrm{m}$ moving with velocity $\mathrm{v}$ has a wave associated with its motion of wavelength

$$
\lambda=\frac{h}{\mathrm{mv}}
$$

and that the total energy of the particle is given by $E=h \nu$, where $v$ is the frequency of the wave associated with the motion of the particle. Then, the time duration of a cycle is

$$
\begin{gathered}
\frac{\lambda}{\mathrm{v}}=\frac{h}{\mathrm{mv}^{2}} \\
\bar{E}=\frac{1}{\lambda / \mathrm{v}} \int_{0}^{\lambda / \mathrm{v}} E_{P} d t=\frac{E_{P} H}{\lambda / \mathrm{v}}=E_{P} H \frac{\mathrm{v}}{\lambda}=E_{P} H \nu,
\end{gathered}
$$

once again yielding $\bar{E}=E$ in the Planck-Einstein equation if $E_{P} H=h$. Note, as $\lambda$ becomes smaller, the energy waveform becomes closer to existing on a larger segment of its cycle, thus reflecting more classical behavior. It is the coming into and out of 4 dim spacetime existence that, we suggest, is the key to understanding nonclassical behavior.

\section{Observations}

(1) The energy waveform $\operatorname{EX}(t)$ is characteristic of a moving particle that comes in and out of view (existence), yet produces waves as a result of its periodic movement through a media.

(2) Instantaneous velocity is a mathematical construct. In practice, it is average velocity (usually over a small time interval) that is determined experimentally. The same applies to instantaneous energy. This is all the more true in the microscopic world where ultimate space, time, and energy are clearly defined by the Planck scale.

(3) In our interpretation of the Planck-Einstein equation, energy of frequency $v$ is delivered over a time shorter than the time wavelength $\lambda / c$, and with energy much higher than $E$. This may help explain how photons pierce the surface of metals, then expel electrons within. It is conceivable that a very small proportion of $E$ does the piercing in the form of a large spike of energy during Planck time duration. 
(4) We may ask, what happens to the energy when the waveform is 0 ? Does conservation of energy still hold? Classically, conservation of energy is time instantaneous; that is, at any instant, the total energy is constant. Thus, we may argue that if a particle has energy at one instant and not in the next, this in itself does not contravene the law of conservation of energy. Secondly, conservation of energy may still be preserved but with respect to a higher-dimensional space. That is, when a photon or particle's energy in 4dimensional space-time vanishes, the energy may actually be transferred to the 6 or 7 additional curled-up space dimensions that string theory [3] purports to exist. In such a scenario total energy would be conserved with respect to a 10- or 11-dimensional space.

(5) The interpretation can be generalized by allowing multiples of $E_{P}$ on different multiple Planck time units as long as the total area under these energy blocks adds up to $h$.

(6) String theory uses Planck energies but in order to end up with realistically low energies, the notion of negative energy is invoked. The foregoing itnerpretation offers a possible alternate mechanism for achieving low energies, namely, time averaging.

\section{Application to the age of the universe}

We distinguish between two times in a particle experiment: (a) the time since the beginning of the experiment, which we refer to as the "age" of the experiment, (b) the total time during the experiment in which the particle actually exists in 4-dimensional spacetime, which we call the "time of existence." It is implicit in this that time runs continuously from the beginning of the experiment, but the particles within 4-dimensional space time come in and out of existence.

It is our objective to correlate the age of the universe and its time of existence based on particles that came into existence at the time of (or close to) the Big Bang. The best candidates for this are the particles of ultra-high energy (UHE) cosmic rays. It was first noted by Greisen [4] and Zatsepin and Kuzmin [8] (GZK) that the upper bound on the spectrum of these rays is $10^{18} \mathrm{eV}$. However, recent experiments [2] have confirmed that the cosmic ray spectrum does not end with the GZK cutoff.

It is known that cosmic rays below $10^{19} \mathrm{eV}$ are created in our galaxy [7]. In [1] it is suggested that these particles are "metastable supermassive relic particles that were created in the early universe and are decaying in the current epoch. The highest end of of the cosmic ray spectrum can thus be used as a probe of new fundamental physics beyond the standard model."

In [6] a new cutoff is predicted in the UHE cosmic ray spectrum of the order $10^{13} \mathrm{GeV}$. As no such particles have yet been discovered we conservatively choose a smaller number for the cutoff: $E=5 \times 10^{12} \mathrm{GeV}$. Now the de Broglie wavelength of a particle is

$$
\lambda=\frac{h}{\mathrm{mv}}=\frac{h}{\left(E / c^{2}\right) \mathrm{v}} .
$$

Since UHE particles move at speeds very close to $c$, we assume $\mathrm{v}=c$. Then we have $\lambda=h c / E$, where $E$ is in Joules, and $\lambda / c=h / E$. From Einstein's formula for conversion of energy to mass, we know that $1 \mathrm{GeV} / \mathrm{c}^{2}=1.783 \times 10^{-27} \mathrm{~kg}$. Thus a particle with energy $10^{12} \mathrm{GeV}$ has mass $=1.783 \times 10^{-15} \mathrm{~kg}$. Using $1 \mathrm{GeV}=1.602 \times 10^{-10}$ Joules, we obtain 
$5 \times 10^{12} \mathrm{GeV}=801 \mathrm{~J}$. Then

$$
\frac{\lambda}{c}=\frac{6.626 \times 10^{-34}}{801}=0.818 \times 10^{-36} \mathrm{sec} .
$$

Since $H=3.39 \times 10^{-43}$ seconds, the fraction of each cycle on which the energy is nonzero, and hence on which the particle exists in 4-dimensional space time is given by

$$
\alpha=\frac{\lambda / c}{H}=2.4 \times 10^{6} .
$$

Assuming that we can regard a UHE particle as having come into existence close to the time of creation, its age is about 14 billion years. But the time it has existed in 4 dimensional space time is a fraction of this time, given by

$$
\frac{14000000000}{2400000}=5833 \text { years. }
$$

Incidentally, this number is in close accordance with the Biblical figure of 5765 years as the total time the universe has existed.

\section{References}

[1] P. Bhattacharjee and G. Sigl, Origin and propagation of extremely high-energy cosmic rays, Physics Reports 327 (2000), no. 3-4, 109-247.

[2] R. P. Feynman, R. B. Leighton, and M. Sands, The Feynman Lectures on Physics. Vol. 3, chapter 37, Addison-Wesley, Massachusetts, 1963.

[3] B. Greene, The Elegant Universe, Vintage, New York, 2000.

[4] K. Greisen, End to the cosmic-ray spectrum?, Physical Review Letters 16 (1966), no. 17, 748-750.

[5] D. Halliday and R. E. Resnick, Fundamentals of Physics, 2nd ed., John Wiley \& Sons, New York, 1981.

[6] V. A. Kuzmin and I. I. Tkachev, Ultrahigh-energy cosmic rays, superheavy long-lived particles, and matter creation after inflation, JETP Letters 68 (1998), no. 4, 271-275.

[7] S. Yoshida, N. Hayashida, K. Honda, M. Honda, S. Imaizumi, N. Inoue, K. Kadota, F. Kakimoto, K. Kamata, S. Kawaguchi, N. Kawasumi, Y. Matsubara, K. Murakami, M. Nagano, H. Ohoka, M. Teshima, I. Tsushima, and H. Yoshii, The cosmic ray energy spectrum above $3 \times 10^{18} \mathrm{eV}$ measured by the Akeno Giant Air Shower Array, Astroparticle Physics 3 (1995), no. 2, 105-123.

[8] G. T. Zatsepin and V. A. Kuzmin, Upper limit of the spectrum of cosmic rays, Pisma v Zhurnal Eksperimentalnoi i Teoreticheskoi Fiziki 4 (1966), 114.

A. Boyarsky: Department of Mathematics and Statistics, Concordia University, Montreal, QC, Canada H4B 1R6

E-mail address: boyar@alcor.concordia.ca 


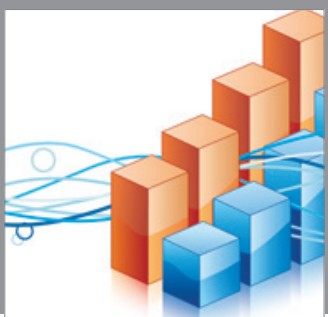

Advances in

Operations Research

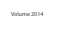

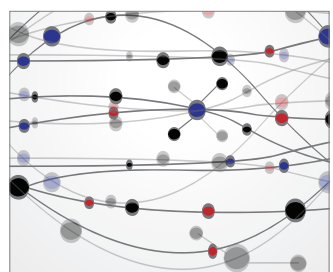

\section{The Scientific} World Journal
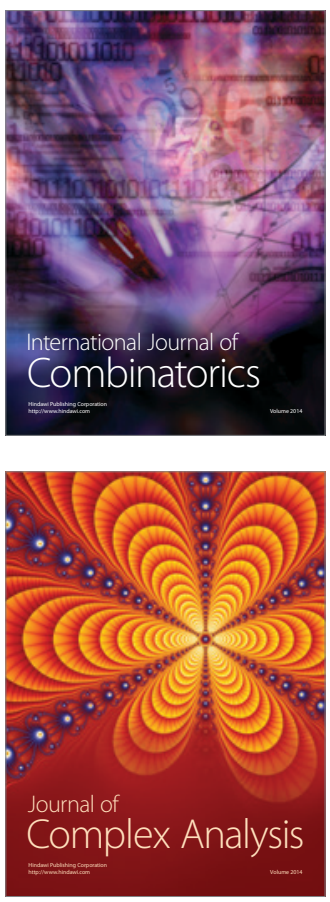

International Journal of

Mathematics and

Mathematical

Sciences
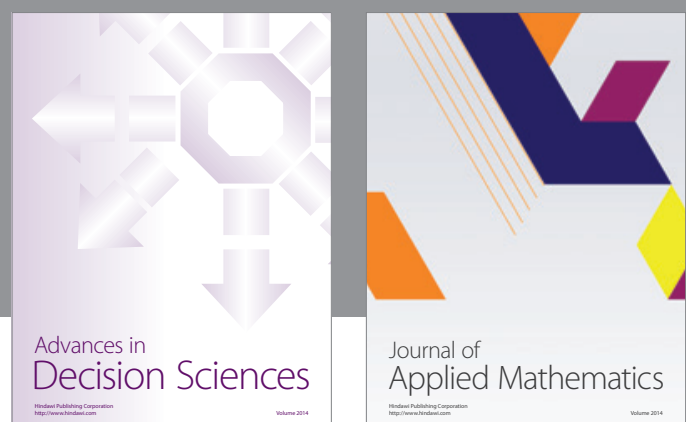

Journal of

Applied Mathematics
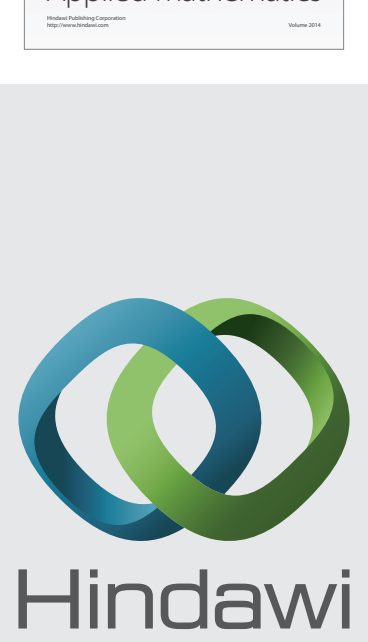

Submit your manuscripts at http://www.hindawi.com
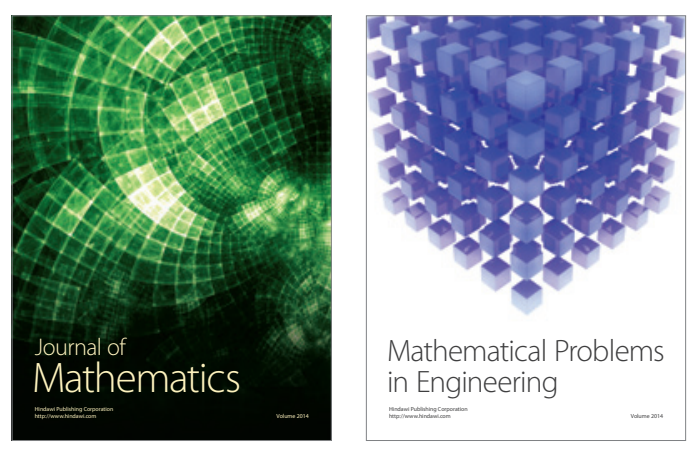

Mathematical Problems in Engineering
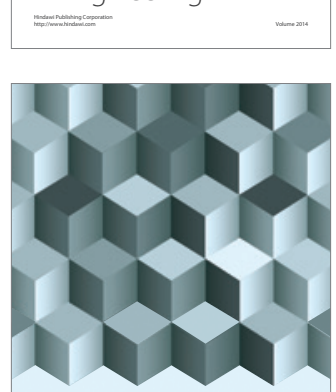

Journal of

Function Spaces
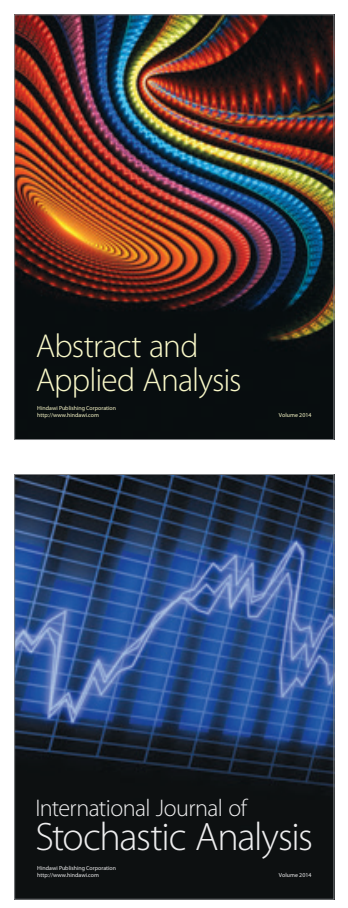

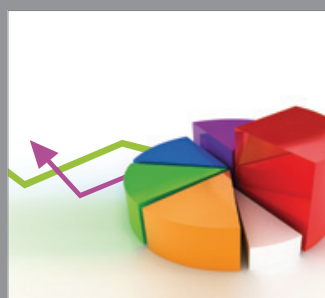

ournal of

Probability and Statistics

Promensencen
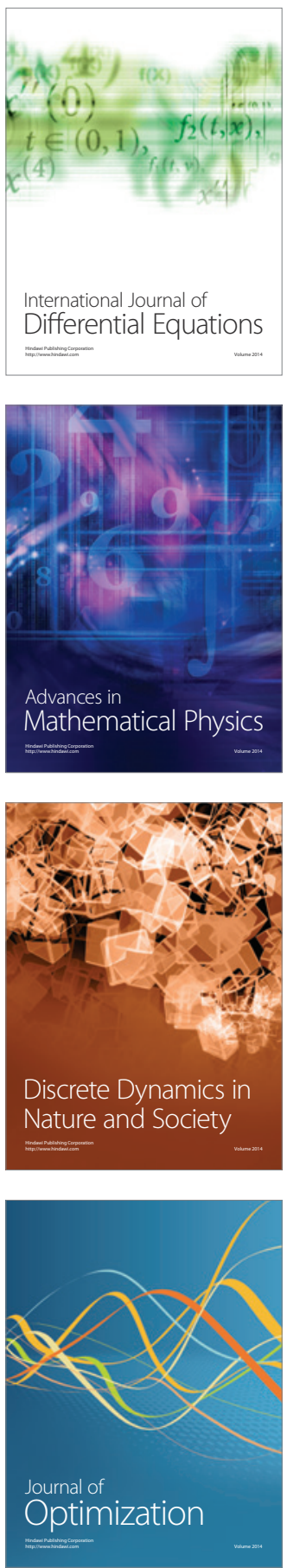